\title{
Untangling Alzheimer's Disease
}

\author{
By Jack M. Gorman, MD
}

There is a story, undoubtedly apocryphal, that two great neuroscientists of the late 19th century, Emil Kraeplin and Alois Alzheimer, struck a bargain. To study dementia, Alzheimer agreed to investigate only the form developing in late-life; Kraeplin investigated cognitive impairment beginning early in life and progressing, what he called "dementia praecox." Alzheimer probably got the better end of the deal. On pathological examination, tangles and plaques were visible throughout the brain in his samples. He described his observations in his only published paper ${ }^{1}$ and Alzheimer's disease became his legacy. Kraeplin, by contrast, saw nothing unusual in his brain samples on postmortem examination and, although he wrote many fine and important papers, there is no "Kraeplin's disease." Schizophrenia, as it is called, turned out to be far less dramatic on microscopic examination than AD.

After 100 years of examining brains, we are no better able to cure AD than schizophrenia. This is frustrating in both cases, but the situation with AD seems particularly peculiar. We have an illness with undeniable, obvious clinical symptoms, a predictable course, and dramatic neuropathololgy. We now know in exquisite detail what constitutes the plaques and tangles found in the brain, we have identified several genes that increase risk for the disease, and we understand some of the most fundamental neurochemical and molecular events responsible for the formation of amyloid and tau protein deposits. And, yet, a cure seems far away. The articles in this month's CNS Spectrums give some indication why this may be and what progress can be made to find better treatments for AD.

Until recently, scientists have concentrated on studying $\mathrm{AD}$ in its most prominent and florid forms, the late stages. Here, the clinical tragedy of $\mathrm{AD}$ is most obvious. The patient has severe dementia and often behavioral disturbance. Even structural magnetic resonance imaging scans show significant atrophy of the cortex. Although the diagnosis cannot be definitively made until postmortem examination, for all intents and purposes a patient at this point in illness progression is fairly easy to diagnose. Scientists have increasingly realized that studying patients at this stage is akin to focusing only on metastatic cancers or end-stage left ventricular failure. That is to say, not much can be done and whatever allowed the patient to reach this irreversible stage is now obscured by overwhelming pathology.

Dr. Gorman is the editor of this jourmal and Esther and Joseph Klingenstein Professor of Psychiatry and chair of the Department of Psychiatry at Mount Sinai School of Medicine in New York City.
Investigators are now struggling to identify the earliest stage of $\mathrm{AD}$, the point at which only mild cognitive disturbance is identifiable. Some are even trying to identify those at risk for $\mathrm{AD}$ in order to get a better sense of the earliest risk factors.

In this issue of CNS Spectrums, distinguished scientists organized by my colleagues Deborah Marin, MD, and Hillel Grossman, MD, at Mount Sinai School of Medicine and the Bronx Veterans Affairs Medical Center in New York City focus a great deal of attention on risk factors and early intervention possibilities. Many of these risk factors, such as diabetes, hypertension, and hyperlipidemia, overlap with those for cardiac disease. These are potentially heartening findings: modifying these factors has been possible, leading to a clear reduction in cardiac disease in the United States. The possibility that this kind of research will lead to important new insights into $A D$ pathogenesis provides hope for prevention. Reading this month's articles, one cannot help but feel optimistic about the future of $\mathrm{AD}$ research. Perhaps these strategies will also extend to even more mysterious central nervous system diseases, so that someday, Kraeplin's disease will not seem quite as formidable either. CNS

\section{REFERENCE}

1. Alzheimer A, Stelzmann RA, Schnitzlein HN, Murtagh FR. An English translation of Alzheimer's 1907 paper, "Uber eine eigenartige Erkankung der Hirnrinde." Clin Anat. 1995;8:429-431.

\section{CALL FOR PAPERS}

CNS Spectrums is requesting submissions of case reports, reviews, and original research articles on a wide variety of neuroscientific clinical topics. Examples of topics include:

- Practical clinical psychiatry

- Neurology and neuropsychiatry in a clinical setting

- Applications of psychopharmacology and pharmacokinetics across the neuropsychiatric spectrum

Especially encouraged are papers covering comorbidities in neurologic disorders (eg, epilepsy with schizophrenia). Other crossover manuscripts geared to deepening the clinician's understanding of disorders and treatments will be given the highest priority.

All submissions are to be sent to Jack M. Gorman, MD, Editor (or, in Europe, to Joseph Zohar, MD, International Editor), c/o MBL Communications, 333 Hudson Street, 7th Floor, New York, NY 10013.

For more information, please see "Author Guidelines" on page 789 . 